\title{
Alien species in South Africa's national parks
}

Dian Spear $^{1 *}$, Melodie A. McGeoch ${ }^{2}$, Llewellyn C. Foxcroft ${ }^{3}$ and Hugo Bezuidenhout ${ }^{4}$

Addresses:

${ }^{1}$ Centre for Invasion Biology, Department of Botany and Zoology, Stellenbosch University, Private Bag X1, Matieland 7602, South Africa.

${ }^{2}$ Centre for Invasion Biology and Cape Research Centre, South African National Parks, P.O. Box 216, Steenberg 7947, South Africa.

${ }^{3}$ Centre for Invasion Biology and Conservation Services, South African National Parks, Private Bag X402, Skukuza 1350, South Africa.

${ }^{4}$ Scientific Services, South African National Parks, P.O. Box 110040, Hadison Park, Kimberley, 8306, South Africa.

*Correspondence: $\underline{\text { dspear@sun.ac.za }}$

Online Checklist. Checklist of alien species in South Africa's 19 national parks. The list is organised by kingdom, class, then family. Common names can be seen in the Online Index. Information sources for the data are provided below the checklist.

National Parks: AE: Addo Elephant, AG: Agulhas, AF: Augrabies Falls, BO: Bontebok, CA: Camdeboo, GG: Golden Gate Highlands, GR: Garden Route,

KG: Kalahari Gemsbok, KA: Karoo, KR: Kruger, MO: Mokala, MP: Mapungubwe, MR: Marakele, MZ: Mountain Zebra, NA: Namaqua, RI: Richtersveld,

TK: Tankwa Karoo, TM: Table Mountain, WC: West Coast

Data sources are divided into publications, grey literature and databases. The specialists and park staff that provided information are listed in the acknowledgements of the paper. Publications include peer-reviewed literature and books. Grey literature includes reports. Databases include the Working for Water Information Management System, online databases such as the Birds in Reserves Project and National Parks databases such as CyberTracker data.

${ }^{\mathrm{SA}}$ South African species, now outside its historical distribution range (extralimital), * Range expanded, ${ }^{\mathrm{BC}}$ Biocontrol agents 


\section{Checklist of alien species in South Africa's 19 national parks}

\begin{tabular}{|c|c|c|c|c|c|c|c|c|c|c|c|c|c|c|c|c|c|c|c|}
\hline & $A E$ & AG & AF & $\mathrm{BO}$ & $\mathrm{CA}$ & GR & GG & $K G$ & $\mathrm{KA}$ & $\mathrm{KR}$ & MP & MR & $\mathrm{MO}$ & $M Z$ & NA & RI & TM & TK & WC \\
\hline Animalia & 24 & 13 & 5 & 15 & 10 & 27 & 12 & 3 & 13 & 48 & 8 & 8 & 7 & 6 & 5 & 6 & 52 & 6 & 21 \\
\hline Actinopterygii & 7 & 4 & & 8 & 2 & 5 & 1 & & & 2 & 1 & 3 & & & & & & & \\
\hline \multicolumn{20}{|l|}{ Centrarchidae } \\
\hline Lepomis macrochirus & & 1 & & 1 & & & & & & & & & & & & & & & \\
\hline Micropterus dolomieu & & & & 1 & & 1 & & & & & & & & & & & & & \\
\hline Micropterus punctulatus & & 1 & & & & & & & & & & & & & & & & & \\
\hline Micropterus salmoides & & 1 & & 1 & & 1 & & & & & & 1 & & & & & & & \\
\hline \multicolumn{20}{|l|}{ Cichlidae } \\
\hline Oreochromis mossambicus ${ }^{S A}$ & 1 & & & & & & & & & & & & & & & & & & \\
\hline Oreochromis niloticus & & & & & & & & & & 1 & 1 & 1 & & & & & & & \\
\hline Tilapia sparrmanii ${ }^{S A}$ & & & & 1 & & & & & & & & & & & & & & & \\
\hline \multicolumn{20}{|l|}{ Clariidae } \\
\hline Clarias gariepinus ${ }^{S A}$ & 1 & & & 1 & 1 & & & & & & & & & & & & & & \\
\hline \multicolumn{20}{|l|}{ Cyprinidae } \\
\hline Cyprinus carpio & 1 & 1 & & 1 & 1 & & 1 & & & 1 & & 1 & & & & & & & \\
\hline Labeo capensis $^{S A}$ & 1 & & & & & & & & & & & & & & & & & & \\
\hline Labeo umbratus $^{S A}$ & 1 & & & & & & & & & & & & & & & & & & \\
\hline Labeobarbus aeneus $^{S A}$ & 1 & & & & & & & & & & & & & & & & & & \\
\hline Tinca tinca & & & & 1 & & & & & & & & & & & & & & & \\
\hline \multicolumn{20}{|l|}{ Poeciliidae } \\
\hline Gambusia affinis & 1 & & & & & 1 & & & & & & & & & & & & & \\
\hline \multicolumn{20}{|l|}{ Salmonidae } \\
\hline Oncorhynchus mykiss & & & & & & 1 & & & & & & & & & & & & & \\
\hline Salmo trutta & & & & 1 & & 1 & & & & & & & & & & & & & \\
\hline Amphibia & & & & & & 1 & & & & & & & & & & & & & \\
\hline \multicolumn{20}{|l|}{ Hyperoliidae } \\
\hline Hyperolius marmoratus ${ }^{S A}$ & & & & & & 1 & & & & & & & & & & & & & \\
\hline \multicolumn{20}{|l|}{ Anthozoa } \\
\hline \multicolumn{20}{|l|}{ Sagartiidae } \\
\hline Sagartia ornata & & & & & & & & & & & & & & & & & & & 1 \\
\hline Arachnida & & & & & & & & & & 3 & & & & & & & & & \\
\hline \multicolumn{20}{|l|}{ Galumnidae } \\
\hline Orthogalumna terebrantis & & & & & & & & & & 1 & & & & & & & & & \\
\hline \multicolumn{20}{|l|}{ Sarcoptidae } \\
\hline Notoedres cati & & & & & & & & & & 1 & & & & & & & & & \\
\hline \multicolumn{20}{|l|}{ Varroidae } \\
\hline Varroa destructor & & & & & & & & & & 1 & & & & & & & & & \\
\hline
\end{tabular}




\begin{tabular}{|c|c|c|c|c|c|c|c|c|c|c|c|c|c|c|c|c|c|c|c|}
\hline \multirow{2}{*}{\multicolumn{20}{|c|}{ Animalia }} \\
\hline & & & & & & & & & & & & & & & & & & & \\
\hline Ascidiacea & & & & & & 3 & & & & & & & & & & & & & 2 \\
\hline \multicolumn{20}{|l|}{ Cionidae } \\
\hline Ciona intestinalis & & & & & & 1 & & & & & & & & & & & & & 1 \\
\hline \multicolumn{20}{|l|}{ Clavelinidae } \\
\hline Clavelina lepadiformis & & & & & & 1 & & & & & & & & & & & & & \\
\hline \multicolumn{20}{|l|}{ Didemnidae } \\
\hline Diplosoma listerianum & & & & & & 1 & & & & & & & & & & & & & 1 \\
\hline Aves & 2 & & 3 & 6 & & 5 & 4 & 2 & 5 & 3 & 3 & 3 & 1 & 3 & 3 & 3 & 7 & 4 & 6 \\
\hline \multicolumn{20}{|l|}{ Anatidae } \\
\hline Anas platyrhynchos & & & & 1 & & 1 & & & & & & & & & & & 1 & & 1 \\
\hline \multicolumn{20}{|l|}{ Columbidae } \\
\hline Columba livia & & & 1 & 1 & & 1 & 1 & 1 & 1 & 1 & 1 & 1 & & 1 & & 1 & 1 & & 1 \\
\hline \multicolumn{20}{|l|}{ Fringillidae } \\
\hline Fringilla coelebs & & & & & & & & & & & & & & & & & 1 & & \\
\hline \multicolumn{20}{|l|}{ Numididae } \\
\hline Numida meleagris ${ }^{S A^{*}}$ & & & & 1 & & 1 & & & 1 & & & & & & 1 & & 1 & 1 & 1 \\
\hline \multicolumn{20}{|l|}{ Passeridae } \\
\hline Passer domesticus & 1 & & 1 & 1 & & 1 & 1 & 1 & 1 & 1 & 1 & 1 & 1 & 1 & 1 & 1 & 1 & 1 & 1 \\
\hline \multicolumn{20}{|l|}{ Phasianidae } \\
\hline Gallus gallus & & & & & & & 1 & & & & & & & & & & & & \\
\hline \multicolumn{20}{|l|}{ Sturnidae } \\
\hline Acridotheres tristis & & & & & & & 1 & & & 1 & 1 & 1 & & & & & & & \\
\hline Sturnus vulgaris & 1 & & & 1 & & 1 & & & 1 & & & & & 1 & & 1 & 1 & 1 & 1 \\
\hline \multicolumn{20}{|l|}{ Threskiornithidae } \\
\hline Bostrychia hagedash ${ }^{S A^{*}}$ & & & 1 & 1 & & & & & 1 & & & & & & 1 & & 1 & 1 & 1 \\
\hline Bivalvia & 1 & & & & & 2 & & & & & & & & & & & & & 1 \\
\hline \multicolumn{20}{|l|}{ Mytilidae } \\
\hline Mytilus galloprovincialis & 1 & & & & & 1 & & & & & & & & & & & & & 1 \\
\hline \multicolumn{20}{|l|}{ Ostreidae } \\
\hline Crassostrea gigas & & & & & & 1 & & & & & & & & & & & & & \\
\hline Chilopoda & & & & & & & & & & & & & & & & & 1 & & \\
\hline \multicolumn{20}{|l|}{ Scutigeridae } \\
\hline Scutigera coleoptrata & & & & & & & & & & & & & & & & & 1 & & \\
\hline Diplopoda & & & & & & & & & & & & & & & & & 2 & & \\
\hline \multicolumn{20}{|l|}{ Julidae } \\
\hline Brachyiulus c.f. pusillus & & & & & & & & & & & & & & & & & 1 & & \\
\hline Ommatoiulus moreletii & & & & & & & & & & & & & & & & & 1 & & \\
\hline
\end{tabular}




\section{Animalia}

$\begin{array}{llllllllllllllllllll}\text { AE } & A G & A F & B O & C A & G R & G G & K G & K A & K R & M P & M R & M O & M Z & N A & R I & T M & T K & \text { WC }\end{array}$

Entognatha

Brachystomellidae

Brachystomella parvula

Hypogastruridae

Hypogastrura armata

Hypogastrura purpurescens

Mesogastrura libyca

Xenylla maritima

Isotomidae

Entomobrya nivalis

Hemisotoma thermophila

Parisotoma notabilis

Proisotoma minuta

Neanuridae

Neanura muscorum

Tomoceridae

Tomocerus minor

\section{Gastropoda}

Agriolimacidae

Deroceras laevae

Deroceras reticulatum

Arionidae

Arion hortensis (aggregate)

Cochlicopidae

Cochlicopa cf. lubricella

Discidae

Discus rotundatus

Gastrodontidae

Zonitoides arboreus

Helicidae

Cornu aspersum

Theba pisana

Limacidae

Deroceras panormitanum

Lehmannia valentiana

Limax maximus

ittorinidae

Littorina saxatilis

Lymnaeidae

Lymnaea columella 


\section{Animalia}

Gastropoda

Oxychilidae

Oxychilus draparnaudi

Physidae

Aplexa marmorata

Physella acuta

Pristilomatidae

Vitrea contracta

Pupilidae

Lauria cylindracea

Thiaridae

Tarebia granifera

Insecta

Agromyzidae

Calcomyza lantanae ${ }^{B C}$

Ophiomyia lantanae ${ }^{B C}$

Anisolabididae

Euborellia annulipes

Apionidae

Trichapion lativentre ${ }^{B C}$

Blaberidae

Nauphoeta cinerea

Pycnoscelus indicus

Blattellidae

Blattella germanica

Blattidae

Periplaneta australasiae

Carposinidae

Carposina autologa ${ }^{B C}$

Cecidomyiidae

Dasineura dielsi ${ }^{B C}$

Cerambycidae

Aphanasium australe ${ }^{B C}$

Chrysomelidae

Leptinotarsa defecta ${ }^{B C}$

Leptinotarsa texana ${ }^{B C}$

Octotoma scabripennis ${ }^{B C}$

Uroplata girard ${ }^{B C}$

Coccinellidae

Harmonia axyridis 


\section{Animalia}

Insecta

Crambidae

Niphograpta albiguttalis ${ }^{B C}$

Pseudopyrausta acutangulalis ${ }^{B C}$

Sameodes albiguttalis ${ }^{B C}$

Curculionidae

Cyrtobagous salviniae ${ }^{B C}$

Erytenna consputa ${ }^{B C}$

Melanterius maculatus ${ }^{B C}$

Melanterius servilus ${ }^{B C}$

Neochetina bruchi ${ }^{B C}$

Neochetina eichhorniae ${ }^{B C}$

Neodiplogrammus quadrivittatus ${ }^{B C}$

Neohydronomus affinis ${ }^{B C}$

Rhyssomatus marginatus ${ }^{B C}$

Dactylopiidae

Dactylopius opuntiae ${ }^{B C}$

Dactylopius tomentosus ${ }^{B C}$

Formicidae

Camponotus rufoglaucus ${ }^{S A}$

Lepisiota incisa ${ }^{S A}$

Linepithema humile

Gracillariidae

Parectopa thalassias ${ }^{B C}$

Gryllidae

Gryllodes supplicans

Lepismatidae

Ctenolepisma longicaudata

Mirid

Eccritotarsus catarinensis ${ }^{B C}$

Falconia intermedia ${ }^{B C}$

Noctuidae

Hypena strigata $^{B C}$

Ortheziidae

Orthezia insignis

Pieridae

Pieris brassicae

Pyralidae

Cactoblastis cactorum $^{B C}$ 


\section{Animalia}

$\begin{array}{llllllllllllllllllll}\text { AE } & A G & A F & B O & C A & G R & G G & K G & K A & K R & M P & M R & M O & M Z & N A & R I & T M & T K & \text { WC }\end{array}$

Insecta

Tingidae

Teleonemia scrupulosa ${ }^{B C}$

Vespidae

Vespula germanica

Malacostraca

Armadillidiidae

Armadillidium vulgare

Porcellionidae

Porcellio scaber

Porcellionides pruinosus

\section{Mammalia}

Bovidae

Aepyceros melampus ${ }^{S A}$

Antidorcas marsupialis ${ }^{S A}$

Bos taurus

Capra hircus

Connochaetes taurinus ${ }^{S A}$

Damaliscus pygargus ${ }^{S A}$

Hemitragus jemlahicus

Hippotragus niger ${ }^{S A}$

Kobus ellipsiprymnus ${ }^{S A}$

Oryx gazella

Ovis aries

Tragelaphus angasii $^{S A}$

Tragelaphus strepsiceros ${ }^{S A}$

Canidae

Canis familiaris

Cervidae

Dama dama

Rusa unicolor

Equidae

Equus asinus

Equus burchelliii

Equus caballus

Felidae

Felis catus

Leporidae

Oryctolagus cuniculus

$\begin{array}{llll}6 & 2 & 1 & 4\end{array}$

1

11

1

1

1

1

1

1
1

28

1

1

1

1

1

11

1

1

1

11 


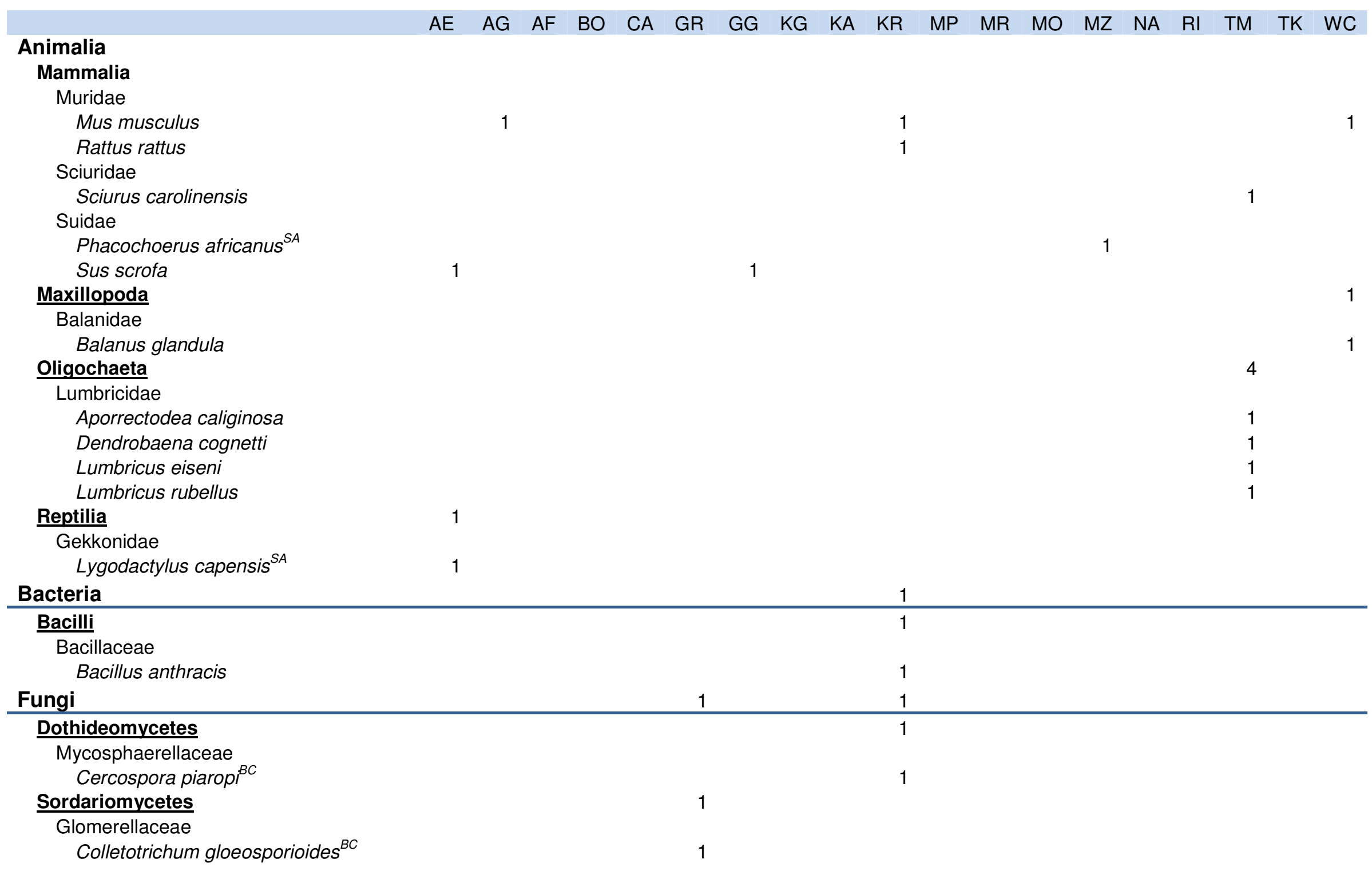




\begin{tabular}{|c|c|c|c|c|c|c|c|c|c|c|c|c|c|c|c|c|c|c|c|}
\hline & $\mathrm{AE}$ & $A G$ & $\mathrm{AF}$ & $\mathrm{BO}$ & CA & GR & GG & $K G$ & KA & $\mathrm{KR}$ & MP & MR & MO & $M Z$ & NA & $\mathrm{RI}$ & TM & TK & WC \\
\hline Plantae & 106 & 75 & 39 & 54 & 44 & 171 & 56 & 12 & 22 & 350 & 37 & 19 & 21 & 69 & 18 & 8 & 239 & 24 & 14 \\
\hline Cycadopsida & & & & & & & & & & 3 & & & & & & & & & \\
\hline \multicolumn{20}{|l|}{ Cycadaceae } \\
\hline Cycas circinalis & & & & & & & & & & 1 & & & & & & & & & \\
\hline Cycas revoluta & & & & & & & & & & 1 & & & & & & & & & \\
\hline \multicolumn{20}{|l|}{ Zamiaceae } \\
\hline Encephalartos ferox ${ }^{S A}$ & & & & & & & & & & 1 & & & & & & & & & \\
\hline Magnoliopsida & 103 & 69 & 38 & 54 & 42 & 162 & 51 & 12 & 22 & 341 & 36 & 19 & 21 & 68 & 18 & 8 & 228 & 24 & 13 \\
\hline \multicolumn{20}{|l|}{ Acanthaceae } \\
\hline Acanthus mollis & & & & & & & & & & 1 & & & & & & & 1 & & \\
\hline Hypoestes phyllostachya & & & & & & & & & & 1 & & & & & & & & & \\
\hline Justicia brandegeeana & & & & & & & & & & 1 & & & & & & & & & \\
\hline Megaskepasma erythrochlamys & & & & & & & & & & 1 & & & & & & & & & \\
\hline Odontonema tubaeforme & & & & & & & & & & 1 & & & & & & & & & \\
\hline Sanchezia oblonga & & & & & & & & & & 1 & & & & & & & & & \\
\hline Strobilanthes dyerianus & & & & & & & & & & 1 & & & & & & & & & \\
\hline Thunbergia alata & & & & & & 1 & & & & 1 & & & & & & & & & \\
\hline \multicolumn{20}{|l|}{ Adoxaceae } \\
\hline Sambucus canadensis & & & & & & 1 & & & & & & & & & & & & & \\
\hline \multicolumn{20}{|l|}{ Agapanthaceae } \\
\hline Agapanthus praecox ${ }^{S A}$ & & & & & & & & & & & & & & & & & 1 & & \\
\hline \multicolumn{20}{|l|}{ Agavaceae } \\
\hline Agave americana & & & & & 1 & & & & & & & & 1 & & 1 & & 1 & 1 & \\
\hline Agave attenuata & & & & & & & & & & 1 & & & & & & & & & \\
\hline Agave sisalana & 1 & 1 & & & 1 & 1 & & & 1 & 1 & 1 & & 1 & 1 & & & 1 & 1 & \\
\hline Agave vivipara & & & & & & & & & & 1 & & & & & & & & & \\
\hline Yucca aloifolia & & & & & & & & & & & & & & & & & 1 & & \\
\hline Yucca gloriosa & & & & & & & & & & & & & & & & & 1 & & \\
\hline \multicolumn{20}{|l|}{ Aizoaceae } \\
\hline Zaleya pentandra ${ }^{S A}$ & & & & & & & & & & 1 & & & & & & & & & \\
\hline \multicolumn{20}{|l|}{ Alliaceae } \\
\hline Allium neapolitanum & & & & & & & & & & 1 & & & & & & & & & \\
\hline Allium triquetrum & & & & & & 1 & & & & & & & & & & & & & \\
\hline Nothoscordum gracile & & & & & & 1 & & & & 1 & & & & & & & & & \\
\hline \multicolumn{20}{|l|}{ Alstroemeriaceae } \\
\hline Alstroemeria pelegrina & & & & & & & & & & 1 & & & & & & & & & \\
\hline \multicolumn{20}{|l|}{ Amaranthaceae } \\
\hline Achyranthes aspera & 1 & & & & & 1 & 1 & & & 1 & 1 & & & & & & 1 & & \\
\hline Alternanthera ficoidea & & & & & & & & & & 1 & & & & & & & & & \\
\hline Alternanthera pungens & & & & & & & & & & 1 & 1 & & & 1 & & & & & \\
\hline Alternanthera sessilis & & & & & & & & & & 1 & & & & & & & & & \\
\hline
\end{tabular}




\section{Plantae}

Amaranthaceae

Amaranthus deflexus

Amaranthus hybridus

Amaranthus spinosus

Amaranthus viridis

Atriplex lindleyi

Atriplex nummularia

Atriplex semibaccata

Chenopodium album

Chenopodium mucronatum

Chenopodium murale

Chenopodium polyspermum

Dysphania ambrosioides

Dysphania carinata

Dysphania schraderiana

Gomphrena celosioides

Guilleminea densa

Hymenocallis littoralis

Iresine herbstii

Pupalia lappacea $^{S A}$

Salsola kali

Anacardiaceae

Harpephyllum caffrum ${ }^{S A}$

Mangifera indica

Rhus lancea ${ }^{S A}$

Schinus molle

Schinus terebinthifolia

1

Annonaceae

Annona reticulata

Apiaceae

Ammi majus

Apium graveolens

Cyclospermum leptophyllum

Foeniculum vulgare

Torilis arvensis ${ }^{S A}$

Apocynaceae

Allamanda cathartica

Araujia sericifera

Asclepias curassavica

Beaumontia grandiflora 


\section{Plantae}

Apocynaceae

Cascabela thevetia

Catharanthus roseus

1

Cryptostegia grandiflora

Gomphocarpus fruticosus

Hoya carnosa

Nerium oleander

Plumeria rubra

Vinca major

Araceae

Anthurium andraeanum

Colocasia esculenta

Epipremnum pinnatum

Monstera deliciosa

Philodendron bipennifolium

Philodendron bipinnatifidum

Pistia stratiotes

Syngonium podophyllum

Araliaceae

Hedera helix

Hydrocotyle americana

Schefflera actinophylla

Schefflera arboricola

Tetrapanax papyrifer

Arecaceae

Archontophoenix cunninghamiana

Caryota mitis

Caryota urens

Chamaedorea elegans

Dypsis lutescens

Howea belmoreana

Livistona chinensis

Phoenix canariensis

Phoenix roebelenii

Ptychosperma macarthurii

Roystonea regia

Sabal palmetto

Syagrus romanzoffiana

Washingtonia robusta 


\section{Plantae}

Aristolochiaceae

Aristolochia littoralis

Asphodelaceae

Aloe arborescens SA

Aloe greatheadii ${ }^{S A}$

\section{Asteraceae}

Acanthospermum australe

Acanthospermum hispidum

Ageratina adenophora

Ageratum conyzoides

Ageratum houstonianum

Anthemis cotula

Aster subulatus

Bidens bipinnata

Bidens biternata

Bidens pilosa

Brachylaena discolor ${ }^{S A}$

Campuloclinium macrocephalum

Carduus tenuiflorus

Centaurea melitensis

Chromolaena odorata

Cirsium vulgare

Conyza albida

Conyza bonariensis

Conyza canadensis

Conyza scabrida ${ }^{S A}$

Conyza sumatrensis

Cosmos bipinnatus

Dittrichia graveolens

Eclipta prostrata

Facelis retusa

Flaveria bidentis

Galinsoga ciliata

Galinsoga parviflora

Gamochaeta purpurea

Helianthus annuus

Hypochoeris brasiliensis

Hypochoeris glabra

Hypochoeris radicata

Lactuca inermis ${ }^{S A}$

1




\section{Plantae}

Asteraceae

Parthenium hysterophorus

Pseudognaphalium luteoalbum

Schkuhria pinnata

Senecio tamoides ${ }^{S A}$

Sonchus asper

Sonchus dregeanus ${ }^{S A}$

Sonchus oleraceus

Sphagneticola trilobata

Symphyotrichum squamatum

Tagetes minuta

Taraxacum officinale

Tithonia diversifolia

Tragopogon dubius

Tridax procumbens

Verbesina encelioides

Xanthium spinosum

Xanthium strumarium

Zinnia peruviana

Balsaminaceae

Impatiens walleriana

Basellaceae

Anredera cordifolia

Basella alba ${ }^{S A}$

Basella paniculata

Berberidaceae

Nandina domestica

Betulaceae

Alnus viridis

Bignoniaceae

Amphilophium buccinatorium

Campsis grandiflora

Dolichandra unguis-cati

Fridericia mollis

Jacaranda mimosifolia

Pyrostegia venusta

Spathodea campanulata

Tabebuia ochracea

Tecoma stans

$\begin{array}{lll}1 & & \\ & & \\ 1 & 1 & \\ 1 & & \\ 1 & & \\ & & \\ & & \\ 1 & 1 & 1 \\ 1 & & \end{array}$


Plantae

Boraginaceae

Echium plantagineum

Echium vulgare

1

Heliotropium indicum

Brassicaceae

Capsella bursa-pastoris

Coronopus integrifolius

Descurainia sophia

Eruca vesicaria

Lepidium bonariense

Lepidium didymum

Lepidium draba

Lepidium ruderale

Nasturtium officinale

Neslia paniculata

Raphanus raphanistrum

Rapistrum rugosum

Sisymbrium orientale

Bromeliaceae

Tillandsia usneoides

\section{Cactaceae}

Austrocylindropuntia cylindrica

Cereus jamacaru

Cylindropuntia fulgida

Cylindropuntia imbricata

Cylindropuntia leptocaulis

Cylindropuntia tunicata

Echinopsis oxygona

Echinopsis spachiana

Harrisia martinii

Hylocereus undatus

Myrtillocactus geometrizans

Opuntia articulata

Opuntia aurantiaca

Opuntia engelmannii

Opuntia ficus-indica

Opuntia humifusa

Opuntia monacantha

Opuntia robusta

Opuntia stricta

1

(1)

1

1 


\section{Plantae}

Cactaceae

Pereskia aculeata

Cannabaceae

Cannabis sativa

Cannaceae

Canna indica

Caricaceae

Carica papaya

Caryophyllaceae

Cerastium capense $^{S A}$

Petrorhagia prolifera

Polycarpaea corymbosa

Polycarpon tetraphyllum

Silene gallica

Spergularia media

Stellaria media

Casuarinaceae

Casuarina cunninghamiana

Cleomaceae

Cleome gynandra

Commelinaceae

Callisia repens

Commelina benghalensis

Dichorisandra thyrsiflora

Tradescantia fluminensis

Tradescantia pallida

Tradescantia spathacea

Tradescantia zebrina

Convolvulaceae

Convolvulus arvensis

Cuscuta campestris

Ipomoea alba

Ipomoea cairica

Ipomoea indica

Ipomoea pes-caprae

Ipomoea purpurea

Ipomoea tricolor

Stictocardia beraviensis

Costaceae

Cheilocostus speciosus 


\section{Plantae}

Crassulaceae

Bryophyllum daigremontianum

Bryophyllum delagoense

Bryophyllum gastonis-bonnieri

Bryophyllum pinnatum

Kalanchoe beharensis

Kalanchoe fedtschenkoi

Cucurbitaceae

Luffa cylindrica

Momordica charantia

Cyperaceae

Cyperus compressus

Cyperus cyperoides

Cyperus esculentus

Cyperus rotundus

Schoenoplectus triqueter

Ericaceae

Erica blenna $^{S A}$

Erica glandulosa ${ }^{S A}$

Erica speciosa

Erica versicolor $^{S A}$

Euphorbiaceae

Acalypha wilkesiana

Codiaeum variegatum

Euphorbia cyathophora

Euphorbia helioscopia

Euphorbia heterophylla

Euphorbia hirta

Euphorbia hypericifolia

Euphorbia inaequilatera

Euphorbia leucocephala

Euphorbia milii

Euphorbia peplus

Euphorbia prostrata

Euphorbia pulcherrima

Euphorbia tithymaloides

Homalanthus populifolius

1

1

1

1

Jatropha podagrica

Manihot esculenta

Ricinus communis 


\section{Plantae}

Fabaceae

Acacia baileyana

Acacia cyclops

Acacia dealbata

Acacia decurrens

Acacia elata

11

Acacia implexa

Acacia longifolia

Acacia mearnsii

Acacia melanoxylon

Acacia paradoxa

Acacia podalyriifolia

Acacia pycnantha

Acacia saligna

Acacia stricta

Adenanthera pavonina

Aeschynomene indica

Bauhinia purpurea

Bauhinia variegata

Caesalpinia decapetala

Caesalpinia gilliesii

Caesalpinia pulcherrima

Cajanus cajan

Calliandra brevipes

Clitoria ternatea

Delonix regia

Erythrina pallida

Genista monspessulana

Gleditsia triacanthos

Gliricidia sepium

Leucaena leucocephala

Lotus subbiflorus

Lupinus angustifolius

Medicago polymorpha

Medicago sativa

Melilotus albus

Melilotus indicus

Mimosa pigra

Ornithopus sativus

Paraserianthes lophantha

$\begin{array}{llll} & 1 & 1 & \\ & & & \\ & & & \\ 1 & 1 & & \\ 1 & 1 & 1 & 1 \\ 1 & & 1 & \\ & & & \\ & 1 & & \\ 1 & 1 & 1\end{array}$

1

1 


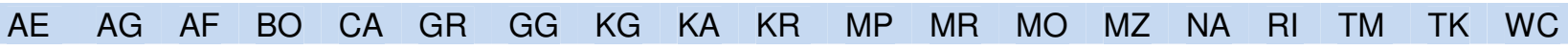

\section{Plantae}

Fabaceae

Parkinsonia aculeata

Prosopis glandulosa

Prosopis velutina

Robinia pseudoacacia

Senna bicapsularis

Senna corymbosa

Senna didymobotrya

Senna multiglandulosa

Senna obtusifolia

Senna occidentalis

Senna pendula

Senna septemtrionalis

Senna spectabilis

Sesbania bispinosa

Sesbania punicea

Spartium junceum

Tipuana tipu

Trifolium angustifolium

Trifolium arvense

Trifolium burchellianum ${ }^{S A}$

Trifolium repens

Trigonella glabra

Vicia benghalensis

Vicia hirsuta

Vicia sativa

$V_{\text {Virgilia divaricata }}^{S A}$

Zornia diphylla

Fagaceae

Castanea sativa

Quercus ilex

Quercus nigra

Quercus palustris

Quercus robur

Quercus suber

Fumariaceae

Fumaria muralis

Geraniaceae

Erodium cicutarium

Erodium moschatum 


\section{Plantae}

Haloragaceae

Myriophyllum aquaticum

Myriophyllum spicatum

Hemerocallidaceae

Dianella tasmanica

Phormium tenax

Hyacinthaceae

Ornithogalum thyrsoides ${ }^{S A}$

Hypericaceae

Hypericum perforatum

Hypoxidaceae

Molineria capitulata

Iridaceae

Iris domestica

Neomarica caerulea

Lamiaceae

Ajuga reptans

Cedronella canariensis

Clerodendrum splendens

Clerodendrum thomsoniae

Lamium amplexicaule

Plectranthus barbatus

Plectranthus scutellarioides

Salvia microphylla

Vitex trifolia

Lauraceae

Cassytha filiformis

Cinnamomum camphora

Litsea glutinosa

Persea americana

Liliaceae

Cordyline fruticosa

ythraceae

Heimia myrtifolia

Lagerstroemia indica

Lythrum salicaria

Malvaceae

Brachychiton populneus

Ceiba pentandra

Corchorus tridens 


\section{Plantae}

Malvaceae

Corchorus trilocularis

Hibiscus rosa-sinensis

Hibiscus schizopetalus

Malva arborea

Malva neglecta

Malva parviflora

Malvastrum coromandelianum

Malvaviscus arboreus

Pachira glabra

Waltheria indica

Marantaceae

Maranta leuconeura

Melastomataceae

Tibouchina elegans

Meliaceae

Melia azedarach

Toona ciliata

Menispermaceae

Cocculus hirsutus ${ }^{S A}$

Molluginaceae

Mollugo nudicaulis

Moraceae

Ficus carica

Morus alba

Moringaceae

Moringa oleifera

Myrtaceae

Callistemon viminalis

Eucalyptus camaldulensis

Eucalyptus camphora

Eucalyptus cinerea

Eucalyptus cladocalyx

Eucalyptus conferruminata

Eucalyptus diversicolor

Eucalyptus globulus

Eucalyptus gomphocephala

Eucalyptus grandis

Eucalyptus lehmannii

Eucalyptus saligna

1 


\section{Plantae}

Myrtaceae

Eucalyptus sideroxylon

Eugenia uniflora

Leptospermum laevigatum

Melaleuca leucadendra

Metrosideros excelsa

Psidium guajava

Syzygium cumini

Syzygium paniculatum

Nyctaginaceae

Boerhavia diffusa

Bougainvillea glabra

Bougainvillea spectabilis

Mirabilis jalapa

Oleaceae

Fraxinus americana

Ligustrum japonicum

Ligustrum sinense

Ligustrum vulgare

Onagraceae

Fuchsia c.f. regia

Oenothera indecora

Oenothera jamesii

Oenothera parodiana

Oenothera rosea

Oenothera stricta

Orchidaceae

Epidendrum obrienianum

Vanilla roscheri

Orobanchaceae

Orobanche minor

Oxalidaceae

Oxalis corniculata

Oxalis latifolia

Pandanaceae

Pandanus tectorius

Pandanus utilis

Papaveraceae

Argemone mexicana

Argemone ochroleuca
1

1

1 


\section{Plantae}

Papaveraceae

Papaver aculeatum ${ }^{S A}$

Passifloraceae

Passiflora caerulea

Passiflora edulis

Passiflora mollissima

Phyllanthaceae

Breynia disticha

Phytolaccaceae

Phytolacca dioica

Phytolacca octandra

Rivina humilis

Piperaceae

Peperomia marmorata

Pittosporaceae

Pittosporum undulatum

Plantaginaceae

Plantago lanceolata

Plantago major

Plantago virginica

Platanaceae

Platanus acerifolia

Plumbaginaceae

Plumbago zeylanica

\section{Poaceae}

Agrostis avenacea

Aira cupaniana

Ammophila arenaria

Arundo donax

Avena fatua

Axonopus fissifolius

Bambusa balcooa

Bambusa multiplex

Bambusa vulgaris

Brachypodium distachyon

Briza maxima

Briza minor

Bromus catharticus

Bromus diandrus

Bromus pectinatus ${ }^{S A}$

1
1

1 


\section{Plantae}

Poaceae

Bromus rigidus

Cenchrus brownii

Cenchrus incertus

Chloris virgata ${ }^{S A}$

Cortaderia jubata

Cortaderia selloana

Cynodon dactylon

Digitaria nuda

Digitaria sanguinalis

Diplotaxis muralis

Echinochloa crus-galli

Echinochloa pyramidalis

Eleusine coracana $^{S A}$

Eragrostis barrelieri

Eragrostis pilosa ${ }^{S A}$

Eragrostis tef

Eragrostis virescens

Hordeum murinum

Hordeum stenostachys

Lagurus ovatus

Lolium multiflorum

Lolium perenne

Lolium rigidum

Lolium temulentum

Melinis repens ${ }^{S A}$

Nasella trichotoma

Panicum maximum ${ }^{S A}$

Panicum schinzii ${ }^{S A}$

Paspalum dilatatum

Paspalum distichum

Paspalum urvillei

Pennisetum clandestinum

Pennisetum purpureum

Pennisetum setaceum

Phalaris arundinacea

Phalaris minor

Poa annua

Poa pratensis

Polypogon monspeliensis

1

1

1

1

1

1

1

1

1

1

$\begin{array}{lllll}1 & 1 & 1 & 1\end{array}$

$\begin{array}{lllll}1 & 1 & 1 & 1 & 1\end{array}$

$\begin{array}{lllll}1 & 1 & 1 & 1 & 1\end{array}$

1

(1)

(1)

(1)




\section{Plantae}

Poaceae

Rostraria pumila

Setaria palmifolia

Setaria parviflora

Setaria verticillata ${ }^{S A}$

Stenotaphrum secundatum

Tragus berteronianus ${ }^{\text {SA }}$

Vulpia bromoides

Vulpia myuros

Polygonaceae

Antigonon leptopus

Emex australis ${ }^{S A}$

Fallopia convolvulus

Muehlenbeckia platyclados

Persicaria lapathifolia

Persicaria limbata

Polygala virgata

Polygonum aviculare

Rumex crispus

Rumex nepalensis

Pontederiaceae

Eichhornia crassipes

Monochoria vaginalis

Portulacaceae

Portulaca oleracea

Portulaca quadrifida ${ }^{S A}$

Talinum paniculatum

Primulaceae

Anagallis arvensis

Proteaceae

Banksia ericifolia

Banksia integrifolia

Grevillea robusta

Hakea gibbosa

Hakea salicifolia

Hakea sericea

Hakea suaveolens

Leucadendron tinctum ${ }^{S A}$

Leucospermum conocarpodendron ${ }^{S A}$

Leucospermum cordifolium ${ }^{S A}$

1

1
1

1

1

1

1

1
1
1

1

$1-1$




\section{Plantae}

Proteaceae

Leucospermum gerrardii ${ }^{S A}$

Leucospermum reflexum ${ }^{S A}$

Protea burchellii ${ }^{S A}$

Protea compacta ${ }^{S A}$

Protea laurifolia ${ }^{S A}$

Protea magnifica $^{S A}$

Protea neriifolia ${ }^{S A}$

Protea susannae ${ }^{S A}$

Stenocarpus sinuatus

Rosaceae

Agrimonia odorata

Cotoneaster franchetii

Cotoneaster pannosus

Duchesnea indica

Eriobotrya japonica

Prunus persica

Pyracantha angustifolia

Pyracantha coccinea

Pyrus communis

Rosa rubiginosa

Rubus affinis

Rubus cuneifolius

Rubus fruticosus

Rubiaceae

Coffea arabica

Gardenia jasminoides

Hamelia chrysantha

Ixora coccinea

Mussaenda frondosa

Richardia brasiliensis

Richardia humistrata

Richardia
Ruscaceae

Aspidistra elatior

Dracaena fragrans

Dracaena reflexa

Liriope muscari

Sansevieria trifasciata

1

1

1

1

1
1

1

1

1

1

Rutaceae

Agathosma betulina $^{S A}$ 


\section{Plantae}

Rutaceae

Agathosma crenulata ${ }^{S A}$

Citrus aurantiifolia

Citrus limon

Citrus sinensis

Salicaceae

Populus alba

Populus canescens

Populus deltoides

Populus nigra

Salix babylonica

Salix fragilis

Sapindaceae

Acer negundo

Cardiospermum grandiflorum

Cardiospermum halicacabum

Litchi chinensis

Scrophulariaceae

Buddleja madagascariensis

Myoporum insulare

Myoporum montanum

Verbascum virgatum

Veronica persica

Simaroubaceae

Ailanthus altissima

\section{Solanaceae}

Browallia americana

Brugmansia arborea

Brunfelsia pauciflora

Capsicum annuum

Cestrum diurnum

Cestrum elegans

Cestrum laevigatum

Datura ferox

Datura inoxia

Datura stramonium

Nicandra physalodes

Nicotiana glauca

Physalis angulata

Physalis peruviana

1

1

1

1
1

1

1

1

1

$\begin{array}{lllllllll}1 & & & & & 1 & & & \\ 1 & & & & \\ 1 & & & & & 1 & 1 & 1 & \\ 1 & 1 & 1 & 1 & 1 & 1 & 1 & & \\ 1 & & & & & 1 & & & \\ 1 & 1 & 1 & & 1 & 1 & & & 1\end{array}$

$\begin{array}{llllllllll}1 & 1 & & 1 & 1 & 1 & & 1 & & 1 \\ 1 & 1 & & & & & 1 & & & \\ 1 & 1 & 1 & 1 & 1 & & & 1 & & \\ 1 & & & & & & & & & \\ 1 & 1 & & & 1 & 1 & 1 & 1 & 1 & 1 \\ 1 & & & & & & & & & \\ 1 & & & & & & & 1 & \end{array}$




\section{Plantae}

Solanaceae

Solanum americanum

1

Solanum betaceum

Solanum chenopodioides

Solanum elaeagnifolium

Solanum laxum

Solanum mauritianum

Solanum physalifolium

Solanum pseudocapsicum

Solanum seaforthianum

Solanum sisymbriifolium

Tamaricaceae

Tamarix chinensis

Tamarix ramosissima

Tamarix usneoides ${ }^{S A}$

Tropaeolaceae

Tropaeolum majus

Urticaceae

Pilea cadierei

Pilea microphylla

Urtica dioica

Urtica membranacea

Urtica urens

Valerianaceae

Centranthus ruber

Verbenaceae

Citharexylum spinosum

Duranta erecta

Glandularia aristigera

Holmskioldia sanguinea

Lantana camara

Stachytarpheta mutabilis

Stachytarpheta urticifolia

Verbena bonariensis

Verbena litoralis

Violaceae

Hybanthus enneaspermus ${ }^{S A}$

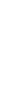

1

1

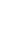

1

11

1

11

$\begin{array}{llll}1 & 1 & 1 & 1\end{array}$

$1-1$

1

1

Cissus antarctica 


\section{Plantae}

Zingiberaceae

Alpinia zerumbet

Hedychium coccineum

Hedychium coronarium

Hedychium gardnerianum

Zingiber zerumbet

Zygophyllaceae

Tribulus terrestris ${ }^{S A}$

\section{Pinopsida}

Cupressaceae

Cryptomeria japonica

Cupressus macrocarpa

Pinaceae

Pinus canariensis

Pinus halepensis

Pinus patula

Pinus pinaster

Pinus pinea

Pinus radiata

Pinus roxburghii

Pinus taeda

Podocarpaceae

\section{Afrocarpus falcatus ${ }^{S A}$}

\section{Pteridopsida}

Aspleniaceae

Asplenium nidus

Azollaceae

Azolla filiculoides

Cyatheaceae

Cyathea australis

Sphaeropteris cooperi

Lomariopsidaceae

Nephrolepis exaltata

Polypodiaceae

Phlebodium aureum

Pteridaceae

Adiantum raddianum

Salviniaceae

Salvinia adnata

16

153

1

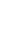

1

1

1
1

2

1

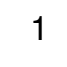

4

2

6

1

1

1

1

1

11

1

1
1

1

1

1

1

1

1

1

1

1

2 


\section{Data sources by park}

\section{Addo Elephant National Park}

Publications:

Branch, W.R. \& Braack, H.H. 1987. Reptiles and amphibians of the Addo Elephant National Park. Koedoe 30, 61-111.

Robinson, T.B., Griffiths, C.L., McQuaid, C.D. \& Rius, M. 2005. Marine alien species of South Africa - status and impacts. African Journal of Marine Science 27, 297-306.

van Wyk, B.E., van Wyk, C.M. \& Novellie, P.A. 1988. Flora of the Zuurberg National Park. 2. An annotated checklist of ferns and seed plants. Bothalia 18, 221 232.

\section{Grey Literature:}

Addo Elephant National Park. 2006. Park Management Plan. October 2006. South African National Parks.

Bezuidenhout, H. 1993. Alfabetiese lys van Addo Nasionale Park. Ongepubliseerde interne verslag. AERU kantore, Kimberley.

Birds of the Addo Elephant National Park. Compiled April 2008 by Marais, C., Bird, J., Patton, C. \& Brown, D.

http://www.sanparks.org/groups/birders/checklists/birds-addo.pdf Accessed 20 September 2010.

Databases: Birds in Reserves Project, CyberTracker data, ISCU Biocontrol Database, Working for Water Information Management System

\section{Agulhas National Park}

Publications:

Rouget, M. \& Richardson, D.M. 2003. Inferring process from pattern in plant invasions: a semimechanistic model incorporating propagule pressure and environmental factors. American Naturalist 162, 713-724.

Russell, I.A. \& Impson, N.D. 2006. Aquatic systems in and adjacent to Agulhas National Park with particular reference to the fish fauna. Koedoe 49 , 45-57.

Theron, J.M., Van Laar, A., Kunneke, A. \& Bredendkamp, B.V. 2004. A preliminary assessment of utilizable biomass in invading Acacia stands on the Cape coastal plains. South African Journal of Science 100, 123-125.

\section{Grey Literature:}

Agulhas National Park. 2008. Park Management Plan. March 2008. South African National Parks.

Avery, G. 1999. Die Kelders Cave 1 (Klipgat). Human Sciences Division, South African Museum, Cape Town.

Bickerton, I.B. 1984. Heuningnes (CSW 19). Report No. 25. In: Heydorn, A.E.F. \& J.R. Grindley (Eds.). Estuaries of the Cape. Part II: Synopsis of available information on individual systems. Council for Scientific and Industrial Research (CSIR Research report no. 424).

Skinner, J.D. \& Smithers, R.H.N. 1990. The mammals of the southern African subregion. University of Pretoria, Pretoria.

Walsh, B.N. 1968. Some notes on the incidence and control of driftsands along the Caledon, Bredasdorp and Riversdale coastline of South Africa. Department of Forestry.

Databases: CyberTracker data, ISCU Biocontrol Database, Working for Water Information Management System 


\section{Augrabies Falls National Park}

\section{Publications:}

Zietsman, P.C. \& Bezuidenhout, H. 1999. Flowering plant biodiversity of Augrabies Falls National Park: a comparison between Augrabies Falls National Park, Kalahari Gemsbok National Park, Vaalbos National Park and Goegap Nature Reserve. Koedoe 42, 95-112.

\section{Grey Literature:}

Augrabies National Park. 2008. Park Management Plan. March 2008. South African National Parks.

Birds of Augrabies Falls National Park. Compiled September 2004. http://www.sanparks.org/groups/birders/checklists/birds-augrabies.pdf Accessed 20 September 2010.

Databases: Birds in Reserves Project, CyberTracker data, Kimberly South African National Park Herbarium, Working for Water Information Management System

\section{Bontebok National Park}

\section{Publications:}

Braack, H.H. 1981. Lower vertebrates of the Bontebok National Park. Koedoe 24, 67-77.

Cambray, J.A. \& Stuart, C.T. 1985. Aspects of the biology of a rare redfin minnow, Barbus burchellii (Pisces, Cyprinidae), from South Africa. South African Journal of Zoology 20, 155-165.

Harrison, A.C. 1963. The Olifants/Doorn River system and its fishing. Piscator 98, 25-28.

Russell, I.A. 2001. Freshwater fishes of Bontebok National Park. Koedoe 44, 71-77.

Winterbottom, J.M. 1967. A preliminary list of the birds of the Bontebok National Park, Swellendam. Koedoe 10, $183-188$.

Grey Literature:

Birds recorded in the Bontebok National Park. http://www.sanparks.org/groups/birders/checklists/birds-bontebok.pdf Accessed 20 September 2010.

Bontebok National Park. 2008. Park Management Plan. March 2008. South African National Parks.

De Moor, J. \& Bruton, M.N. 1988. Atlas of alien and translocated indigenous aquatic animals in southern Africa. South African National Scientific Programmes Report 144. Council for Scientific and Industrial Research, Pretoria.

Databases: Birds in Reserves Project, Working for Water Information Management System

\section{Camdeboo National Park}

Publications:

Masubelele, M.L., Foxcroft, L.C. \& Milton, S.J. 2009. Alien plant species list and distribution for Camdeboo National Park, Eastern Cape Province, South Africa. Koedoe 51, 80-89.

Grey Literature:

Appendix 4 - Lower level plan for Camdeboo National Park's rehabilitation programme. South African National Parks.

Camdeboo National Park. 2008. Park Management Plan. March 2008. South African National Parks.

Databases: CyberTracker data, ISCU Biocontrol Database, Working for Water Information Management System 


\section{Garden Route National Park}

\section{Publications:}

Coates, T.J. 1970. Checklist of the Collembola of South African parks (Part 1), Koedoe 13, 181-184

Robinson, T.B., Griffiths, C.L., McQuaid, C.D. \& Rius, M. 2005. Marine alien species of South Africa - status and impacts. African Journal of Marine Science 27, 297-306.

Russell, I.A. 1999. Freshwater fish of the Wilderness National Park. Koedoe 42, 73-78.

Russell, I.A. 2002. Freshwater fishes of Tsitsikamma National Park. Koedoe 45, 13-17.

Skead, C.J. \& Liversidge, R. 1967. Birds of the Tsitsikamma forest and coastal national park, 1966. Koedoe 10, 43-62.

Grey Literature:

Bird's of Tsitsikamma National Park. Compiled September 2004. http://www.sanparks.org/groups/birders/checklists/birds-tsitsikamma.pdf Accessed 20 September 2010.

Calvo-Ugarteburu, G. 1998. The influence of environmental factors and disease on the biology and productivity of the Pacific Oyster Crassostrea gigas in the Knysna Estuary. Project Report, Department of Zoology and Entomology, Rhodes University, Grahamstown. 77pp plus appendices.

De Moor, J. \& Bruton, M.N. 1988. Atlas of alien and translocated indigenous aquatic animals in southern Africa. South African National Scientific Programmes Report 144. Council for Scientific and Industrial Research, Pretoria.

Skelton, P.H. 1993. A complete guide to the freshwater fishes of southern Africa. Southern Book Publishers, Halfway House.

Tsitsikamma National Park. 2008. Park Management Plan. March 2008. South African National Parks.

Wilderness National Park. 2008. Park Management Plan. March 2008. South African National Parks.

Databases: Birds in Reserves Project, ISCU Biocontrol Database, Working for Water Information Management System

\section{Golden Gate Highlands National Park}

Publications:

Russell, I.A. \& Skelton, P.H. 2005. Freshwater fishes of Golden Gate Highlands National Park. Koedoe 48, 87-94.

Grey Literature:

Appendix 4 - Lower level plan for Golden Gate Highlands National Park's Rehabilitation Programme. South African National Parks.

Birds of Golden Gate Highlands National Park. Compiled September 2004. http://www.sanparks.org/groups/birders/checklists/birds-goldengate.pdf Accessed 20 September 2010.

Gaylard, A. \& Taljaard, J. 2008. Golden Gate National Park - Park Management Plan. March 2008. South African National Parks.

Databases: Birds in Reserves Project, CyberTracker data, Kimberly South African National Park Herbarium, Working for Water Information Management System

\section{Kalahari Gemsbok National Park}

Publications:

van Rooyen, N. \& Bezuidenhout, H. 1997. New records of flowering plants and ferns from the Kalahari Gemsbok National Park. Koedoe 40, $105-116$.

Grey Literature:

Birds of Kgalagadi Transfrontier Park. September 2004. http://www.sanparks.org/groups/birders/checklists/birds-kgalagadi.pdf Accessed 20 September 2010.

Kalahari Gemsbok National Park. 2008. Park Management Plan. March 2008. South African National Parks.

Databases: Birds in Reserves Project, CyberTracker data, Kimberly South African National Park Herbarium 


\section{Karoo National Park}

\section{Grey Literature}

Birds of Karoo National Park. Compiled September 2004. http://www.sanparks.org/groups/birders/checklists/birds-karoo.pdf Accessed 20 September 2010.

Gaylard, A. \& Johnson, N. 2008. Karoo National Park - Park Management Plan. March 2008. South African National Parks.

Databases: Birds in Reserves Project, CyberTracker data, Kimberly South African National Park Herbarium, Working for Water Information Management System

\section{Kruger National Park}

\section{Publications:}

Braack, L.E.O., Maggs, K.A.R., Zeller, D.A. \& Horak, I.G. 1995. Exotic arthropods in the Kruger National Park, South Africa: modes of entry and population status. African Entomology 3, 39-48.

Coates, T.J. 1970. Checklist of the Collembola of South African parks (Part 1), Koedoe 13, 181-184.

De Kock, K.N. \& Wolmarans, C.T. 1998. A re-evaluation of the occurrence of freshwater mollusks in the Kruger National Park. Koedoe 41, 1-8.

De Kock, K.N. \& Wolmarans, C.T. 2007. Distribution and habitats of the alien invader freshwater snail Physa acuta in South Africa. Water SA 33, 717-722.

De Kock, K.N. \& Wolmarans, C.T. 2008. Invasive alien freshwater snail species in the Kruger National Park, South Africa. Koedoe 50, $49-53$.

Foxcroft, L.C., Henderson, L., Nichols, G.R. \& Martin, B.W. 2003. A revised list of alien plants for the Kruger National Park. Koedoe 46, 21-44.

Herbert, D.G. 2010. The introduced terrestrial Mollusca of South Africa. SANBI Biodiversity Series 15. South African National Biodiversity Institute, Pretoria.

MacDonald, I.A.W. \& Frame, G.W. 1988. The invasion of introduced species into nature reserves in tropical savannas and dry woodlands. Biological Conservation 44, 67-93.

MacDonald, I.A.W. \& Gertenbach, W.P.D. 1998. A list of alien plants in the Kruger National Park. Koedoe 31, 137-150.

Grey Literature:

Biggs, H., Freitag-Ronaldson, S., Eber, S. \& Venter, F. 2008. Kruger National Park - Park Management Plan. March 2008. South African National Parks.

Complete checklist of birds of Kruger National Park. Compiled August 2008 by Chris Patton. http://www.sanparks.org/groups/birders/checklists/birds-

kruger comprehensive 0810.pdf Accessed 20 September 2010.

Kruger National Park. Invasive alien species operational programme: plants. South African National Parks.

Databases: Birds in Reserves Project, ISCU Biocontrol Database, Working for Water Information Management System

\section{Mapungubwe National Park}

Publications:

Gotze, A.R., Cilliers, S.S., Bezuidenhout, H \& Kellner, K. 2008. Analysis of the vegetation of the sandstone ridges (IB land type) of the north-eastern parts of the Mapungubwe National Park, Limpopo Province, South Africa. Koedoe 50, 72-81.

Grey Literature:

Biggs, H., Freitag-Ronaldson, S. \& Nemaheni, T. 2008. Mapungubwe National Park - Park Management Plan. March 2008. South African National Parks.

Birders checklist for the Mapungubwe National Park and area. Compiled by Etienne Marais. http://www.sanparks.org/groups/birders/checklists/birds-

mapungubwe.pdf Accessed 20 September 2010.

Foxcroft, L. 2006. Mapungubwe National Park. Invasive alien species plan. June 2006. South African National Parks.

Databases: CyberTracker data, Kimberly South African National Park Herbarium, Working for Water Information Management System 


\section{Marakele National Park}

\section{Grey Literature:}

Birds of Marakele National Park. http://www.sanparks.org/groups/birders/checklists/birds-marakele.pdf Accessed 20 September 2010.

Foxcroft, L. 2006. Marakele National Park. Invasive alien species plan. June 2006. South African National Parks.

Freitag-Ronaldson, S. Trennery, C. \& Mugwabana, F.H. 2008. Marakele National Park - Park Management Plan. March 2008. South African National Parks.

Databases: CyberTracker data, Kimberly South African National Park Herbarium

\section{Mokala National Park}

Grey Literature:

Daemane, E. \& Spies, A. 2008. Mokala National Park - Park Management Plan. June 2008. South African National Parks.

Databases: Birds in Reserves Project, CyberTracker data, Working for Water Information Management System

\section{Mountain Zebra National Park}

\section{Publications:}

Bezuidenhout, H. \& Brown, L.R. 2008. Vegetation description of the Doornhoek section of the Mountain Zebra National Park (MZNP), South Africa. Koedoe 50, 8292.

Pond, U., Beesley, B.B., Brown, L.R. \& Bezuidenhout, H. 2002. Floristic analysis of the Mountain Zebra National Park, Eastern Cape. Koedoe 45, 35-57.

\section{Grey Literature:}

Birds-MountainZebra.pdf http://www.sanparks.org/groups/birders/checklists/birds-mountainzebra.pdf Accessed 20 September 2010.

Gaylard, A., Mjadu, M. \& de Klerk, J. 2008. Mountain Zebra National Park - Park Management Plan. March 2008. South African National Parks.

Novellie, P. 1988. Mountain Zebra National Park Management Plan. South African National Parks.

Databases: Birds in Reserves Project, CyberTracker data, Kimberly South African National Park Herbarium, Working for Water Information Management System

\section{Namaqua National Park}

\section{Grey Literature:}

Bird checklist for Namaqua National Park. Compiled March 2006 by Japie Claassen. http://www.sanparks.org/groups/birders/checklists/birds-namaqua.pdf Accessed 20 September 2010

Namaqua National Park. 2009. Lower level alien plant clearing plan. Draft 1 of 2009. South African National Parks.

Namaqua National Park. 2008. Park Management Plan. March 2008. South African National Parks.

Databases: CyberTracker data, Working for Water Information Management System 


\section{Richtersveld National Park}

\section{Publications:}

Hendricks, H.H., Novellie, P.A., Bond, W.J. \& Midgley, J.J. 2002. Diet selection of goats in the communally grazed Richtersveld National Park. African Journal of Range and Forage Science 19, 1-11.

Grey Literature:

Birds of Richtersveld National Park. Compiled September 2004. http://www.sanparks.org/groups/birders/checklists/birds-richtersveld.pdf Accessed 20 September 2010.

Richtersveld National Park. 2009. Lower level alien plant clearing plan. Draft 1 of 2009. South African National Parks.

Databases: CyberTracker data, Kimberly South African National Park Herbarium

\section{Table Mountain National Park}

Publications:

Alston, K.P. \& Richardson, D.M. 2006. The roles of habitat features, disturbance, and distance from putative source populations in structuring alien plant invasions at the urban/wildland interface on the Cape Peninsula, South Africa. Biological Conservation 132, 183-198.

Coates, T.J. 1970. Checklist of the Collembola of South African parks (Part 1), Koedoe 13, 181-184.

Herbert, D.G. 2010. The introduced terrestrial Mollusca of South Africa. SANBI Biodiversity Series 15. South African National Biodiversity Institute, Pretoria.

Pryke, J.S. \& Samways, M.J. 2008. Conservation of invertebrate biodiversity on a mountain in a global biodiversity hotspot, Cape Floral Region. Biodiversity and Conservation 17, 3027-3034.

\section{Grey Literature:}

Adamson, R.S. \& Salter, T.M. 1950. Flora of the Cape Peninsula. Juta, Cape Town.

Birds of Table Mountain National Park. Compiled September 2004. http://www.sanparks.org/groups/birders/checklists/birds-tablemountain.pdf Accessed 20

September 2010.

Moll, E.J. \& Scott, L. 1981. Trees and shrubs of the Cape Penninsula. Eco-lab Trust Fund, University of Cape Town, Rondebosch

Raharinjanahary, D. 2007. Impact of Argentine ants (Linepithema humile Mayr) on saproxylic invertebrates in Afromontane forest and pine plantation of the Cape Peninsula (South Africa). Percy FitzPatrick Institute, University of Cape Town, Cape Town.

Siegfried, W.R. 1962. Introduced vertebrates in the Cape Province. Cape Department of Nature Conservation Annual Report 19, 80-87.

Table Mountain National Park - Central. 2009. Lower level alien plant clearing plan. Draft 1 of 2009. South African National Parks.

Table Mountain National Park - North. 2009. Lower level alien plant clearing plan. Draft 1 of 2009. South African National Parks.

Table Mountain National Park - South. 2009. Lower level alien plant clearing plan. Draft 1 of 2009. South African National Parks.

Table Mountain National Park. 2008. Park Management Plan. March 2008. South African National Parks.

Databases: Birds in Reserves Project, ISCU Biocontrol Database, Working for Water Information Management System 


\section{Tankwa National Park}

\section{Publications:}

Rubin, F. 1998. The physical environment and major plant communities of the Tankwa-Karoo National Park. Koedoe 41, 61-94.

Grey Literature:

Bird Checklist for Tankwa Karoo National Park. Compiled July 2007 by Japie Classen. http://www.sanparks.org/groups/birders/checklists/birds-tankwa.pdf

Accessed 20 September 2010.

Freitag-Ronaldson, S., Strauss, C. \& Coetsee, L. 2008. Tankwa Karoo National Park - Park Management Plan. March 2008. South African National Parks.

Tankwa Karoo National Park. 2009. Lower level alien plant clearing plan. Draft 1 of 2009. South African National Parks.

Databases: Birds in Reserves Project, Working for Water Information Management System

\section{West Coast National Park}

Publications:

Avery, D.M., Rautenbach, I.L. \& Randall, R.M. 1990. An annotated check list of the land mammal fauna of the West Coast National Park. Koedoe 33, 1-18.

Laird, M.C. \& Griffiths, C.L. 2008. Present distribution and abundance of the introduced barnacle Balanus glandula Darwin in South Africa. African Journal of Marine Science 30, 93-100.

Odendaal, L.J., Haupt, T.M. \& Griffiths, C.L. 2008. The alien invasive land snail Theba pisana in the West Coast National Park: is there cause for concern? Koedoe 50, 93-98.

Robinson, T.B., Griffiths, C.L. \& Kruger, N. 2004. Distribution and status of marine invasive species in and bordering the West Coast National Park. Koedoe 47, 79-87.

Robinson, T.B., Griffiths, C.L., McQuaid, C.D. \& Rius, M. 2005. Marine alien species of South Africa - status and impacts. African Journal of Marine Science 27, 297-306.

\section{Grey Literature:}

Birds of West Coast National Park. September 2004. http://www.sanparks.org/groups/birders/checklists/birds-westcoast.pdf Accessed 20 September 2010.

West Coast National Park. 2008. Park Management Plan. March 2008. South African National Parks.

West Coast National Park. 2009. Lower level alien plant clearing plan. Draft 1 of 2009. South African National Parks.

\section{Databases: Birds in Reserves Project, Working for Water Information Management System}

